\title{
Digital Therapeutics for Obesity and Eating-Related Problems
}

\author{
Meelim $\mathrm{Kim}^{1,2}$, Hyung Jin Choi ${ }^{1,2}$ \\ ${ }^{1}$ Department of Biomedical Sciences, ${ }^{2}$ BK21 Plus Biomedical Science Project Team, Seoul National University College of \\ Medicine, Seoul, Korea
}

In recent years, digital technologies have rapidly advanced and are being applied to remedy medical problems. These technologies allow us to monitor and manage our physical and mental health in our daily lives. Since lifestyle modification is the cornerstone of the management of obesity and eating behavior problems, digital therapeutics (DTx) represent a powerful and easily accessible treatment modality. This review discusses the critical issues to consider for enhancing the efficacy of DTx in future development initiatives. To competently adapt and expand public access to DTx, it is important for various stakeholders, including health professionals, patients, and guardians, to collaborate with other industry partners and policy-makers in the ecosystem.

Keywords: Digital healthcare; Obesity; Feeding behavior

\section{INTRODUCTION}

Digital therapeutics (DTx) is "an evidence-based therapeutic intervention using high-quality software programs to prevent, manage, or treat a medical disorder or disease" [1]. DTx emerged as a novel therapeutic approach for the prevention, management, or treatment of chronic, behavior-changeable diseases in recent years. Due to the public health emergency posed by coronavirus disease 2019, the Food and Drug Administration (FDA) has relaxed its regulations to expand access to digital health devices for remote monitoring and management of illness [2]. Currently, DTx products target behavior-modifiable problems such as type 2 diabetes and weight management. For example, Welldoc Communications, a system to provide a mobile phone-based diabetes management software connected to web-based data analytics, serves as an interactive platform for patients and healthcare providers that provides real-time information and analysis [3]. There are also other digital healthcare companies for diabetes and weight control; Noom, Omada, Livongo, Lark, Voluntis, and so on. In this review, we discuss evidence-based therapeutic interventions driven by high-quality software programs. Only telephone calls, short message services, or online web-based servers are excluded from the definition of DTx.

Mostly, DTx systems draw upon the evidence-based principles of behavioral or psychological intervention protocols known as cognitive behavioral therapy (CBT) [2]. CBT uses evidence-based techniques to change problematic behaviors and unhelpful cognitive distortions, and to improve emotional regulation and coping skills to solve the current problems [4]. Frequent clinical assessments in the context of diverse psychological conditions using digital modalities are highly beneficial to
Received: 26 January 2021, Revised: 10 February 2021,

Accepted: 17 February 2021

Corresponding author: Hyung Jin Choi

Department of Biomedical Sciences, Seoul National University College of Medicine, 103 Daehak-ro, Jongno-gu, Seoul 03080, Korea

Tel: +82-2-740-8204, Fax: +82-2-745-9528, E-mail: hjchoi@snu.ac.kr
Copyright $\odot 2021$ Korean Endocrine Society

This is an Open Access article distributed under the terms of the Creative Commons Attribution Non-Commercial License (https://creativecommons.org/ licenses/by-nc/4.0/) which permits unrestricted non-commercial use, distribution, and reproduction in any medium, provided the original work is properly cited. 
enhance the efficacy of CBT interventions [5]. In fact, collecting assessment data in face-to-face conditions is a burdensome task involving retrospective reports. These reports have high risks of being systematically biased by recall and neglecting important contextual components or acute changes in certain periods [5]. In contrast, collecting assessment data using smartphones and wearables is convenient and time-sensitive, allowing the real-time evaluation of context-rich information. Thus, the integration of digital technologies and CBT techniques has become the cutting-edge approach in DTx components, allowing individualized and stepped-care interventions [6].

\section{DTx FOR OBESITY AND EATING-RELATED PROBLEMS: AN EMPHASIS ON A MULTIDISCIPLINARY APPROACH}

Since obesity and eating behavioral problems are complex diseases with a multifactorial etiology, a biopsychosocial approach including medical treatment and lifestyle changes is required to treat them effectively [7]. As shown in Fig. 1, both physical and mental health conditions are the major components of lifestyle modification. To achieve a healthy lifestyle through a lifestyle modification intervention, high motivation is mandatory as a prerequisite for high adherence. A patient can also attain stimulus control capability by manipulating eating-related cues in the environment, using cognitive techniques to investigate mal- adaptive thinking, and building coping skills related to emotional regulation and stress management [8]. These psychological mechanisms contributing to mental health are closely associated with physical health and contribute to clinical outcomes.

Mental health should be considered a vital component of DTx for obesity and eating-related problems. However, most previous self-management DTx studies were limited by only focusing on behavioral aspects apart from mental health components, such as self-monitoring of glucose and body weight, medication adherence, logging a food diary, and physical activity $[9,10]$. The majority of mobile health interventions only showed effective improvements in primary outcomes for physical health measures, such as hemoglobin A1c or body weight [11-14]. These interventions were ineffective at improving mental health conditions such as self-efficacy, quality of life, depression, and other measures. We recently showed that motivation, depression, anxiety, and self-esteem were predictive markers of successful weight control [15]. Thus, a multidisciplinary approach including psychological components is needed to develop practical DTx for obesity and other eating behavioral problems.

\section{RECENT RANDOMIZED CONTROLLED TRIALS RELATED TO DTX}

To encourage an informed discourse about the efficacy of DTx for obesity and eating-related problems, we review several ran-

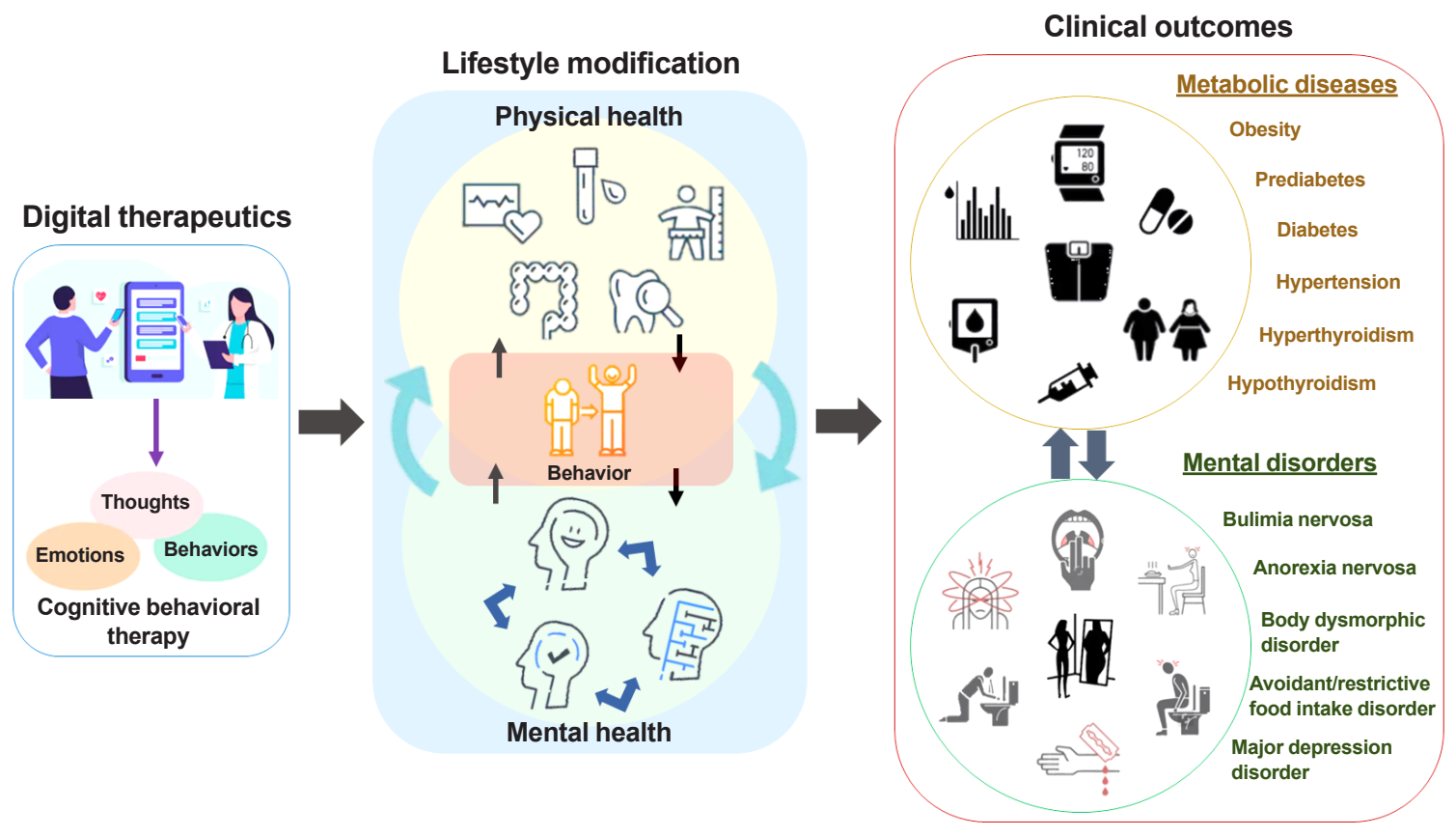

Fig. 1. Interaction between mental and physical health for lifestyle modification via digital therapeutics. 


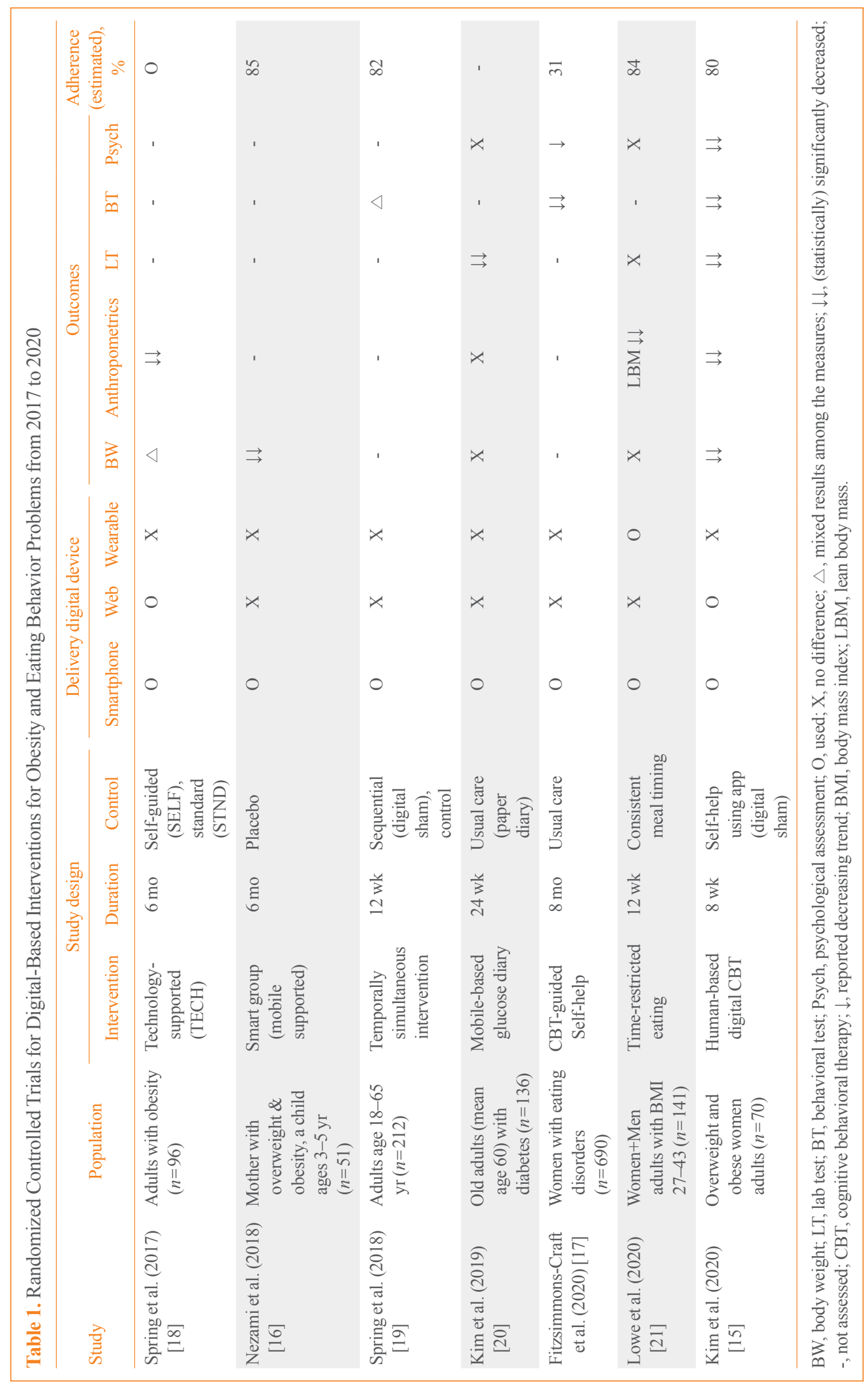


domized controlled trials (RCTs) of DTx conducted from 2017 to 2020 (Table 1).

Regarding the study population, only one DTx RCT was conducted among young children [16]. In fact, the intervention was not directly delivered to the children, but supported their caregivers to reduce sugar-sweetened beverage intake. In terms of the target diseases, four RCTs investigated DTx for obesity treatment, while only one study focused on eating disorders [17].

Two studies applied psychological evidence-based strategies (i.e., CBT) in their intervention protocols, considering cognitions, emotions, and behaviors for lifestyle modification [15,17]. Other studies utilized only behavioral strategies such as selfmonitoring or restricting time [16,18-21]. To test the efficacy of the interventions, two studies used a three-arm parallel-group design [18,19], while other studies used a two-arm parallelgroup design [15-17,20,21].

Regarding the digital devices utilized in the research, three studies combined two different modalities (i.e., smartphone+ web or smartphone+wearable) to deliver the intervention [15, 19,21]. All studies applied smartphones as their core intervention devices [15-21].
Although obesity and eating behavioral problems are known to have complex backgrounds, most studies evaluated clinical efficacy with only one or two measures [16-19]. A few studies applied several assessments to validate their intervention efficacy, but they did not show significant outcomes in general [20,21]. Our study is the only RCT that assessed all five relevant dimensions: body weight, body composition, physiological tests, behavioral tests, and psychological assessments [15]. All clinical outcomes significantly improved after the digital intervention period.

It is also important to evaluate the adherence rate of an intervention, since adherence is a key component for understanding the clinical efficacy of DTx. An engagement rate of over $80 \%$ was shown in most studies $[16,19,21]$. However, the only study that adapted self-help strategies in the digital intervention presented an adherence rate of $31 \%$ [17].

\section{MAIN ISSUES RELATED TO DTx FOR OBESITY AND EATING BEHAVIOR PROBLEMS}

Based on the current status of the field, we suggest seven major

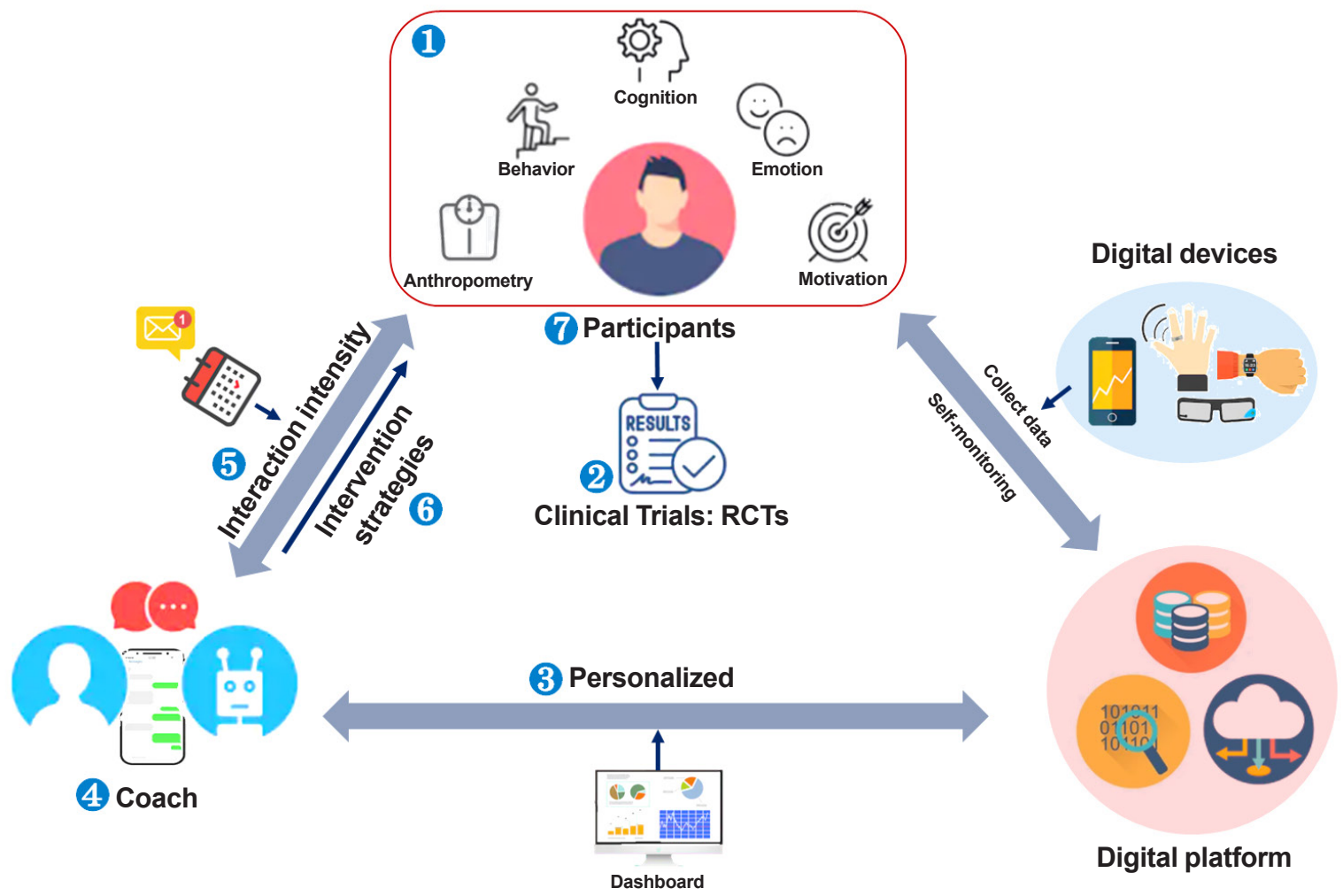

Fig. 2. Major considerations and main issues for digital therapeutics. RCT, randomized controlled trial. 
constructive issues that should be addressed in order to make progress in DTx for obesity and eating-related problems (Fig. 2).

\section{Comprehensiveness of an individual's multifactorial health condition}

As mentioned earlier, obesity and eating behavior problems have complicated contexts [8]. Therefore, multifactorial domains - behavioral (e.g., late-night meals, snacks), cognitive (e.g., arbitrary inference, selective abstraction), emotional (e.g., irritation, loneliness), motivational (i.e., willingness to change, self-confidence), and anthropometric — are suggested to be addressed to comprehensively understand and monitor the progress of weight control and eating behavioral patterns [15]. Since cognitive structuring and emotional regulation significantly affect behavior styles and engagement with DTx, an integrated approach concerned with all of these components will ultimately solve problems related to obesity.

\section{Efficacy of DTx in RCTs}

The aim of RCTs is to test the effectiveness of new treatments while minimizing biases (e.g., the placebo effect). RCTs prove both efficacy and safety, which are decisive components related to FDA approval. Although numerous studies have tested digital interventions for weight loss, not all of them were designed as RCTs [22]. To reliably prove the efficacy of novel DTx, RCTs are a prerequisite.

A critical and challenging aspect of RCT design for DTx is designing an adequate control group. Digital modalities have a variety of features (e.g., self-monitoring, CBT, user interface/ user experience, human coaching, etc.), which results in ambiguity regarding which features are effective, and which specific features should be compared [23]. Thus, it is challenging to select the main active ingredient of DTx and devise a specific active sham control group (identical DTx platform without the main active ingredient). It is also difficult to establish a completely blinded condition, as in placebo-controlled trials of medications. Therefore, new RCT frameworks tailored to DTx have been developed; such as the multiphase optimization strategy (MOST), sequential multiple assignment randomized trials (SMART), micro-randomized trials, clustered randomized control trials, unequal-allocation RCTs, and control optimization trials [24-27]. Each design aims to answer different research questions and thereby to provide the gold standard for proof in clinical medicine. In addition, due to the nature of digital technologies, RCTs of DTx could adopt fully digital online innovative designs, including digital enrollment, digital intervention, and digital outcome phenotyping, potentially avoiding the requirement for on-site visits.

\section{Tailoring the individual feedback in DTx}

Personalized DTx can deliver tailored feedback based on personal data from multifactorial domains, as mentioned above in the discussion of the first issue. We have recently shown that this is critical since personalization is closely related to engagement in digital intervention and the potential for lifestyle change in the long-term [15]. However, most app-based interventions use non-tailored behavioral strategies (e.g., prompts for monitoring, appointment reminders, and health education), uniformly produced common content, or limitedly customized algorithms based on a few domains such as diet, physical activity, and body weight $[17,20]$. These strategies have shown limitations regarding engagement in the intervention and the maintenance of treatment efficacy. Thus, based on baseline and/or real-time multifactorial measures (e.g., behavior, emotion, cognition, and motivation), tailored feedback and adaptive intervention can increase the engagement and the effectiveness of DTx. Furthermore, other characteristics such as, genetic, social, and economic factors, as well as comorbidities, could be utilized for the development of tailored DTx.

\section{The role of a health coach}

A health coach is a person who delivers an evidence-based intervention to users. Embedding a human factor, such as healthcare providers or peers, may enhance the engagement and efficacy of DTx. Recently, we have shown better clinical outcomes from interventions with a human coach compared to those with self-guided conditions $[15,17,18]$. The major role of a health coach is to provide practical solutions to problems, emotional support, motivational interviews, and informative knowledge to support effective behavioral changes. The function of human coaching can be automated by virtual conversational agents, such as chatbots. Natural language processing studies could help develop augmented text coaching platforms for both human coaches and chatbots.

\section{Temporal strategies for intervention frequency}

Temporal strategies for intervention frequency are an important factor influencing the engagement rate in DTx. Broadly, there are three types of time points for an intervention: daily, weekly, and monthly. Previous studies applying either weekly or monthly interventions showed high attrition rates [28]. In contrast, we recently showed that more intensive daily coaching could pro- 
duce a high adherence rate, with $80 \%$ of participants remaining active users until the last session of the treatment. A reason for this high adherence rate is that we delivered more intensive daily personalized feedback by facilitating real-time access to a human coach. Since the engagement rate in DTx influences the clinical outcomes, considering the intensity of interaction between providers and users is necessary. More intensive daily interventions could stimulate higher engagement. However, an extremely frequent intervention could be fatiguing or burdensome to both users and coaches. This can be alleviated by implementing advanced digital technologies such as artificial intelligence or machine-learning approaches to replace repetitive tasks by automated services [29].

\section{Evidence-based psychological theory for intervention strategies}

Although digital health modalities have become very well-designed, the extent to which they involve evidence-based behavior change strategies or clinical protocols should be examined. Evidence-based interventions are defined as intervention strategies with empirical support for their efficacy and accountability. There are several representative psychological interventions; CBT, dialectical behavior therapy, acceptance and commitment therapy, and mindfulness-based cognitive therapy. CBT is widely applied to various types of mental health conditions. Thus, many researchers are exploring possibilities to expand its applicability, especially using digital modalities. Adopting these scientifically proven intervention strategies for behavioral change will produce more efficacious DTx. Furthermore, it is recommended to involve health professionals (i.e., doctors, nurses, psychologists, and trainers) in the development process of DTx for interventions to be reliable.

\section{Target populations}

Most DTx related to lifestyle modifications or eating-related problems have focused on the adult population. Although the epidemic of childhood/adolescent obesity is responsible for the prevalence of metabolic diseases in adults, studies of DTx for lifestyle modifications targeting youth are scarce. To treat children or young adults, it is more efficient to employ their caregivers/guardians in the intervention to achieve efficacious clinical outcomes. A similar point holds for older adults, who are more likely to already have metabolic or psychiatric diseases. It is recommended to include their families in the intervention to obtain successful outcomes via DTx. Furthermore, clinicians may consider implementing applicable strategies according to the target symptoms, such as major depressive disorder, eating disorders, diabetes, or hypertension.

\section{LIMITATIONS OF DTx BEING APPLIED IN THE CLINICS}

To date, many efforts have been made to develop various types of DTx, but still they remain unsettled in the clinical settings due to following limitations. Currently, low engagement and low efficacy of DTx are two major concerns in understanding the full scalability and competency of it [28]. Next, similar to traditional pharmaceuticals, DTx must undergo RCTs for the approval procedure to verify safety and efficacy in the premarket [2]. However, DTx software is capable of being frequently updated and adjusted to FDA guidance at any moment, unlike other pharmaceuticals. Lastly, cybersecurity and data rights are other bottlenecks of the mass adoption of DTx. One of the solutions can be the partnerships between the industries and academics, which the companies behind successful DTx collaborate with academic groups to address scientific rigor as expected of traditional pharmaceutical drugs.

\section{FUTURE PERSPECTIVES AND RECOMMENDATIONS}

As the field of behavioral medicine is leveraging digital technologies to heighten the scalability and effectiveness of interventions, we would like to make the following recommendations for future DTx researchers and industries (Fig. 3).

\section{Different phenotyping methods with multi-dimensional phenotypes}

Applying various phenotyping methods to gather individual information is required to optimize DTx [15]. Phenotypes can be classified as digital or conventional. The digital phenotype refers to both passively and actively gathered data using digital modalities, such as smartphones and wearables [30]. It can be acquired by wearable sensors, smartphones, and other digital devices. The conventional phenotype refers to measures that are assessed by traditional methods (i.e., blood samples, self-reported questionnaires, electronic medical records, and buffet testmeals). These collected phenotypes are then classified into multi-dimensional phenotypes (physical health, behavioral, cognitive, emotional, and motivational). In other words, each of these dimensions can be assessed by either (or both) of these two phenotyping methods. A comprehensive integration of con- 


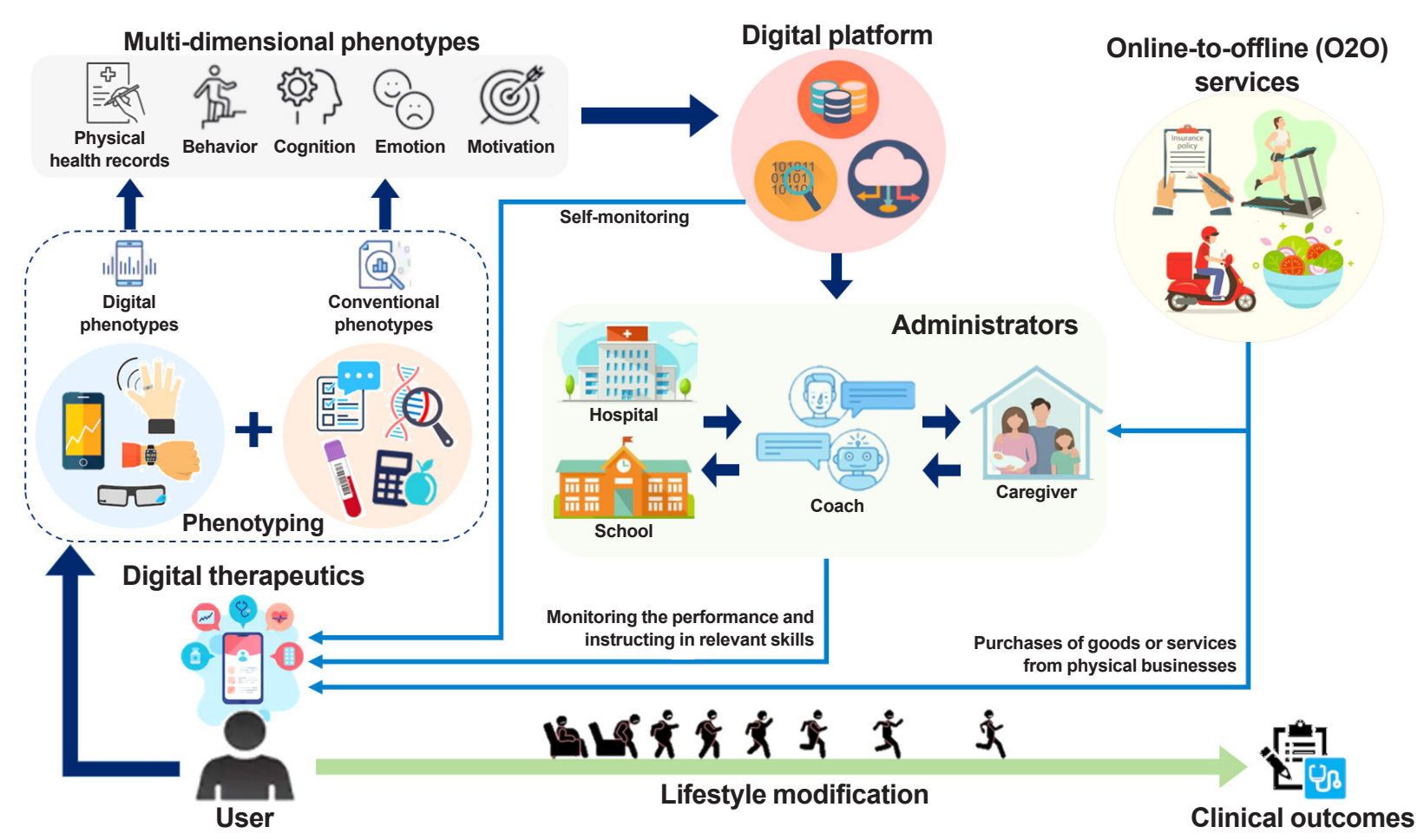

Fig. 3. Future perspectives for the ecological environment of digital therapeutics.

ventional and digital phenotypes is a key component for developing and delivering personalized interventions within DTx [15].

\section{Devising analytical methods and developing dashboards for administrators}

Regarding analytical methods, we suggest applying artificial intelligence technologies and machine-learning analyses to extract clinically meaningful features from immense and intricate data to obtain useful insights for clinical decision-making. It is then important to create insightful visualizations displayed on dashboards for administrators including coaches, caregivers, school teachers, and health professionals, as well as the users themselves [31]. Here, the term "administrators" refers to the people who monitor individuals' performance and instruct them about relevant skills to promote lifestyle modifications. Developing different versions of dashboards for each administrator may generate a better user environment since administrators could then support users in diverse aspects. This will improve individuals' performance, leading to better clinical outcomes.

\section{Integrating DTx with online-to-offline services}

When DTx is delivered to individuals from administrators, its efficacy can be expanded by combining online-to-offline $(\mathrm{O} 2 \mathrm{O})$ services with the DTx intervention. For example, providing discounted passes to work out in a gym would be beneficial for people who need to reduce their body fat and build muscle strength. Another possibility would be enabling people to purchase fitness equipment (e.g., a treadmill or barbell) on sale by bundling these services with DTx. Moreover, delivering fresh salads every day would be applicable for people who find it challenging to prepare healthy meals. Expanding these systems may allow us to establish a ubiquitous environment (i.e., smart homes or smart schools) that adapt advanced digital technologies in daily life. Hospitals, workplaces, schools, homes and healthcare-related markets will be seamlessly connected via DTx $[32,33]$. All administrators will be able to prescribe homecare, work-care, school-care, and medical-care-related products through DTx. As boundaries between different sectors are fading away, new policies are required for stakeholders including health professions, patients, and guardians. This will enable sustainable ecosystems and business models that serve the public's interest.

\section{CONCLUSIONS}

Advanced digital technologies have been leveraged to behavioral medicine, establishing "DTx." DTx provides new opportu- 
nities to easily access effective interventions in daily lives. Recent studies showed that DTx can be applied to other endocrinological diseases such as diabetes, thyroid disease, and sarcopenia $[34,35]$. To develop efficient DTx for obesity and eating problems, it is required to monitor and manage both physical and mental health conditions. Thus, to facilitate the response to lifestyle modification, obesity-related phenotypes including the clusters of behavioral, emotional, cognitive, motivational, and physiological features are required. Since adherence is the strongest predictors for successful weight management, we recommend adjusting a health coach system, providing tailored feedback. Moreover, adequate RCT with active placebo, evidence-based psychological theory (i.e., CBT), and intervention temporal strategies are other important issues to be considered in developing DTx. We are living in the digital era where digital transformation is inevitable. To adapt to this forthcoming flow, new policy actions are necessary in the community, city, government, and industry.

\section{CONFLICTS OF INTEREST}

No potential conflict of interest relevant to this article was reported.

\section{ACKNOWLEDGMENTS}

This study was supported by a National Research Foundation of Korea (NRF) grant funded by the Korean government (MIST) (No. NRF-2018R1A5A2025964).

\section{ORCID}

Meelim Kim https://orcid.org/0000-0002-0507-1727

Hyung Jin Choi https://orcid.org/0000-0003-0593-6978

\section{REFERENCES}

1. Digital Therapeutics Alliance. A new category of medicine [Internet]. Arlington: Digital Therapeutics Alliance; 2020 [cited $2021 \mathrm{Feb} 23$ ]. Available from: https://dtxalliance.org.

2. Patel NA, Butte AJ. Characteristics and challenges of the clinical pipeline of digital therapeutics. NPJ Digit Med 2020;3:159.

3. Quinn CC, Shardell MD, Terrin ML, Barr EA, Ballew SH, Gruber-Baldini AL. Cluster-randomized trial of a mobile phone personalized behavioral intervention for blood glu- cose control. Diabetes Care 2011;34:1934-42.

4. Murphy R, Straebler S, Cooper Z, Fairburn CG. Cognitive behavioral therapy for eating disorders. Psychiatr Clin North Am 2010;33:611-27.

5. Wilhelm S, Weingarden H, Ladis I, Braddick V, Shin J, Jacobson NC. Cognitive-behavioral therapy in the digital age: presidential address. Behav Ther 2020;51:1-14.

6. Lattie EG, Schueller SM, Sargent E, Stiles-Shields C, Tomasino KN, Corden ME, et al. Uptake and usage of IntelliCare: a publicly available suite of mental health and wellbeing apps. Internet Interv 2016;4:152-8.

7. Castelnuovo G, Pietrabissa G, Manzoni GM, Cattivelli R, Rossi A, Novelli M, et al. Cognitive behavioral therapy to aid weight loss in obese patients: current perspectives. Psychol Res Behav Manag 2017;10:165-73.

8. Wadden TA, Tronieri JS, Butryn ML. Lifestyle modification approaches for the treatment of obesity in adults. Am Psychol 2020;75:235-51.

9. Garabedian LF, Ross-Degnan D, Wharam JF. Mobile phone and smartphone technologies for diabetes care and selfmanagement. Curr Diab Rep 2015;15:109.

10. Holtz B, Lauckner C. Diabetes management via mobile phones: a systematic review. Telemed J E Health 2012;18:17584.

11. Ku EJ, Park JI, Jeon HJ, Oh T, Choi HJ. Clinical efficacy and plausibility of a smartphone-based integrated online real-time diabetes care system via glucose and diet data management: a pilot study. Intern Med J 2020;50:1524-32.

12. Guo H, Zhang Y, Li P, Zhou P, Chen LM, Li SY. Evaluating the effects of mobile health intervention on weight management, glycemic control and pregnancy outcomes in patients with gestational diabetes mellitus. J Endocrinol Invest 2019; 42:709-14.

13. Quinn CC, Shardell MD, Terrin ML, Barr EA, Park D, Shaikh F, et al. Mobile diabetes intervention for glycemic control in 45- to 64-year-old persons with type 2 diabetes. $\mathrm{J}$ Appl Gerontol 2016;35:227-43.

14. Kirwan M, Vandelanotte C, Fenning A, Duncan MJ. Diabetes self-management smartphone application for adults with type 1 diabetes: randomized controlled trial. J Med Internet Res 2013;15:e235.

15. Kim M, Kim Y, Go Y, Lee S, Na M, Lee Y, et al. Multidimensional cognitive behavioral therapy for obesity applied by psychologists using a digital platform: open-label randomized controlled trial. JMIR Mhealth Uhealth 2020;8: e14817. 
16. Nezami BT, Ward DS, Lytle LA, Ennett ST, Tate DF. A $\mathrm{mHealth}$ randomized controlled trial to reduce sugar-sweetened beverage intake in preschool-aged children. Pediatr Obes 2018;13:668-76.

17. Fitzsimmons-Craft EE, Taylor CB, Graham AK, SadehSharvit S, Balantekin KN, Eichen DM, et al. Effectiveness of a digital cognitive behavior therapy-guided self-help intervention for eating disorders in college women: a cluster randomized clinical trial. JAMA Netw Open 2020;3:e2015633.

18. Spring B, Pellegrini CA, Pfammatter A, Duncan JM, Pictor A, McFadden $\mathrm{HG}$, et al. Effects of an abbreviated obesity intervention supported by mobile technology: the ENGAGED randomized clinical trial. Obesity (Silver Spring) 2017;25: 1191-8.

19. Spring B, Pellegrini C, McFadden HG, Pfammatter AF, Stump TK, Siddique J, et al. Multicomponent mHealth intervention for large, sustained change in multiple diet and activity risk behaviors: the Make Better Choices 2 randomized controlled trial. J Med Internet Res 2018;20:e10528.

20. Kim EK, Kwak SH, Jung HS, Koo BK, Moon MK, Lim S, et al. The effect of a smartphone-based, patient-centered diabetes care system in patients with type 2 diabetes: a randomized, controlled trial for 24 weeks. Diabetes Care 2019;42:3-9.

21. Lowe DA, Wu N, Rohdin-Bibby L, Moore AH, Kelly N, Liu YE, et al. Effects of time-restricted eating on weight loss and other metabolic parameters in women and men with overweight and obesity: the TREAT randomized clinical trial. JAMA Intern Med 2020;180:1-9.

22. Levine BJ, Close KL, Gabbay RA. Reviewing U.S. connected diabetes care: the newest member of the team. Diabetes Technol Ther 2020;22:1-9.

23. Arigo D, Jake-Schoffman DE, Wolin K, Beckjord E, Hekler EB, Pagoto SL. The history and future of digital health in the field of behavioral medicine. J Behav Med 2019;42:6783.

24. Chakraborty B, Collins LM, Strecher VJ, Murphy SA. Developing multicomponent interventions using fractional factorial designs. Stat Med 2009;28:2687-708.

25. Collins LM, Murphy SA, Strecher V. The multiphase optimization strategy (MOST) and the sequential multiple as- signment randomized trial (SMART): new methods for more potent eHealth interventions. Am J Prev Med 2007;32 (5 Suppl):S112-8.

26. Hekler EB, Rivera DE, Martin CA, Phatak SS, Freigoun MT, Korinek E, et al. Tutorial for using control systems engineering to optimize adaptive mobile health interventions. J Med Internet Res 2018;20:e214.

27. Liao P, Klasnja P, Tewari A, Murphy SA. Sample size calculations for micro-randomized trials in mHealth. Stat Med 2016;35:1944-71.

28. Torous J, Michalak EE, O'Brien HL. Digital health and engagement-looking behind the measures and methods. JAMA Netw Open 2020;3:e2010918.

29. Kavakiotis I, Tsave O, Salifoglou A, Maglaveras N, Vlahavas I, Chouvarda I. Machine learning and data mining methods in diabetes research. Comput Struct Biotechnol J 2017;15:10416.

30. Jain SH, Powers BW, Hawkins JB, Brownstein JS. The digital phenotype. Nat Biotechnol 2015;33:462-3.

31. Lee KH, Yoo S, Shin H, Baek RM, Chung CY, Hwang H. Development of digital dashboard system for medical practice: maximizing efficiency of medical information retrieval and communication. Stud Health Technol Inform 2013;192:1091.

32. Lindberg J, Bhatt R, Ferm A. Older people and rural eHealth: perceptions of caring relations and their effects on engagement in digital primary health care. Scand J Caring Sci 2021 Jan 14 [Epub]. https://doi.org/10.1111/scs.12953.

33. Kang HY, Kim HR. Impact of blended learning on learning outcomes in the public healthcare education course: a review of flipped classroom with team-based learning. BMC Med Educ 2021;21:78.

34. Lee JE, Lee DH, Oh TJ, Kim KM, Choi SH, Lim S, et al. Clinical feasibility of monitoring resting heart rate using a wearable activity tracker in patients with thyrotoxicosis: prospective longitudinal observational study. JMIR Mhealth Uhealth 2018;6:e159.

35. Addante F, Gaetani F, Patrono L, Sancarlo D, Sergi I, Vergari G. An innovative AAL system based on IoT technologies for patients with sarcopenia. Sensors (Basel) 2019;19:4951. 